\title{
VOLUME CHARACTERISTICS OF MAGNETIC-CHANNEL FLARES
}

\author{
L. Kִ̆ IVSKÝ \\ Astronomical Institute, Czechoslovak Academy of Sciences, Ondřejov, Czechoslovakia
}

\begin{abstract}
On the basis of $\mathrm{H} \alpha$ pictures of two proton flares of medium size, the volume of the separate parts of magnetic-channel flares, are given. At the time of the main release of energy the volume of the whole system is determined at $\sim 2.5 \times 10^{27} \mathrm{~cm}^{3}$ and the size of areas III after Petschek model at $\sim 7.0 \times 10^{20} \mathrm{~cm}^{3}$.
\end{abstract}

\section{Areas of the Origin of Accelerated Particles in Flares}

Vaiana et al. (1968) found from pictures of the flare of $8 / 6 / 1968$, importance $1 \mathrm{~N}$ in the $\mathrm{X}$-ray emission 3.5-14 $\AA$, that the volume in which this two-ribbon flare, connected at the narrowest point by a loop coupling, radiated, amounted to $10^{28} \mathrm{~cm}^{3}$; this was roughly at the time of maximum brightness of the flare in $\mathrm{H} \alpha$. The determination of the volume is based on the permissible assumption that the ribbons of the flare have a circular cross-section diameter equal to the width of the ribbons measured on the photographs. It is necessary to point out that the flare was in an active region, where a proton flare with the usual typical development for this type of flare, occurred a day later, on 9/6/1968: two parting emission ribbons, a weaker diffusion ribbon in the emission in the center between them being generated, and an absorption filament (Křivský and Švestka, 1969) coming from the mouth of this flare channel. The flare of $8 / 6 / 1968$, recorded in the X-ray emission by Vaiana et al., therefore, was only one of the precursors of the later typical proton flare, and it was of a kind which does not differ much from the mighty, fully developed proton flares (Křivský, 1963, 1970). Provided considerations are made along the lines of Vaiana et al. (1968), i.e. that the emission areas indicate the space where the particles are accelerated (which, it seems, are identical with this type of flare to volumes 'enveloped' by layers radiating in $\mathrm{H} \alpha$ ) the volumes one obtains at the beginning of the development of proton flares, when the main emission of energy occurs in the form of accelerated particles, will be sufficiently representative for determining the volumes where the principal acceleration of the particles occurs.

If one accepts the fact that in the case of the flare observed in the X-ray emission by Vaiana et al. (1968), the processes in the three coordinate components, inherent to mighty proton flares (Křivský, 1968, 1970), had already developed at least to a minor extent, this is proof that the accelerating processes are basically of two types and that they are located in two volumes, differing in shape: (1) in two flare quasi-parallel ribbons, located horizontally with respect to the surface of the Sun, and (2) in an arched space, vertically developed between both flare ribbons. These two types of volumes can easily be distinguished at first glance on photographs of the flare in the X-ray emission of 8/6/1968 (Vaiana et al., 1968, Figure 2A; Neupert, 1969, Figure 11; Vaiana, 1969). The determination of these two fundamental volumes for accelerating 
the particles, along the neutral lines (in the flare ribbons) on the one hand, and in the magnetically closed, generating structure of the vertically developing loop tunnel with the peak emission ribbon, formed the contents of the model of proton flares published earlier. This model is founded on the interaction of an emerging magnetic channel with a magnetic system of spot groups (Křivský, 1968, 1970).

It will not be an error, at least as far as the order is concerned, if the characteristic volumes for the initial rapid particle acceleration of an impulse nature (at the time of the $Y$-phase), as well as for the later development of the acceleration system in the volumes of the higher ascending parts of the flare channel, will be derived from the photographs of proton flare in $\mathrm{H} \alpha$ (Křivský, 1969); this is substantiated by the conclusions in the paper of Vaiana et al. (1968) on the identity in shape and position of the flare ribbons of the $\mathrm{X}$ - and $\mathrm{H} \alpha$-emissions.

The fundamental volume characteristics of the individual phases of proton flares of an intermediate size, as well as of their physically differing volumes, have been determined from two flares, i.e. the disc flare of $12 / 7 / 1961$ and the limb flare of 18/11/1968 (Křivský, 1970). Also the critical volume for the narrow regions of the microturbulent layers, important for accelerating the particles according to the Petschek wave model, (denoted in Petschek's model as regions III, 1964), was determined.

\section{Volume Characteristics of Proton Flares}

The determination of volumes of flares with an emission of fast particles has been the subject of a number of papers. For example, De Jager (1967) for all types of hard Xemission gives the volume $10^{25}-10^{28} \mathrm{~cm}^{3}$; in (1969) he proved theoretically that the active flare plasma comes from a volume of $10^{29} \mathrm{~cm}^{3}$. For 'optical' flares he gives the volume of just under $10^{26} \mathrm{~cm}^{3}$, and high-energy particles are considered to come from a volume of $10^{27} \mathrm{~cm}^{3}$. Vaiana et al. (1968), using the photographs of the X-ray emission flare of $8 / 6 / 1968$, give a volume of $10^{28} \mathrm{~cm}^{3}$. Friedman and Hamberger (1969) determined theoretically the volume of slow stationary shock fronts of regions III (according to Petschek) in which the magnetic energy transforms to plasma energy accompanied by a microwave emission as $10^{19}-10^{20} \mathrm{~cm}^{3}$. In (Křivský, 1969) the volumes of the proton flare of 26/9/1963 were determined by measuring the photographs in $\mathrm{H} \alpha$ at various stages of development: at the beginning during the $Y$-phase as $10^{26}-10^{27} \mathrm{~cm}^{3}$, and less for the hard X-ray emission; the initial volume of the ribbon as the peak of the loops about $10^{26} \mathrm{~cm}^{3}$, later $10^{28} \mathrm{~cm}^{3}$; the volume of the whole channel of a perfectly developed flare as $10^{29}-10^{30} \mathrm{~cm}^{3}$. Takakura (1969) has fixed the volume as $10^{29} \mathrm{~cm}^{3}$, in which at the explosive phase, a hot coronal condensation $\left(10^{7}-10^{8} \mathrm{~K}\right)$ with hard X-ray bursts, originated. Švestka (1970) determined the volume of the region of origin of a hard X-ray burst as $5 \times 10^{27} \mathrm{~cm}^{3}$ and of a hot condensation at the peak of development as about $10^{29} \mathrm{~cm}^{3}$. Important results are also in Zirin (1964) and in Acton (1968).

With a view to the known phase of development of proton flares, which are called magnetic-channel flares (Křivský, 1963, 1968, 1969a, b, 1970) and which have also 
been described in a later phase of their development by Bruzek and DeMastus (1970). Also for a number of reasons following from the time connections of the phases of development with the $\gamma$-emission, with the $\mathrm{X}$-ray emission, with the radio emission, and with white-light radiation, it is necessary to divide the measured and computed volume charateristics of this type of flare into three stages:

Stage I - $Y$-phase (beginning of the splitting of the flare ribbon into two), at the time of the occurrence of the hard and very hard impulsive X-ray emission (Krivský in Švestka, 1966; Valníček, 1967; Křivský and Švestka, 1969), at the time of the first radio burst (mm, cm) and at the time of the integral flare-emission (Śvestka, 1970); this phase at the very beginning of the flare is considered to be a process in which the main transformation of the magnetic energy in the fast particle flux takes place (cosmic and subcosmic radiation).

Stage II - approximately at the time of the maximum flare brightness. This stage was originally considered by a number of authors to be the phase in which the very fast particles are emitted (e.g., Ellison et al., 1961). It seems that this stage is characteristic for the generation and emission of a cloud of plasma, or for the escape of particles, accelerated to a lesser extent in the peak regions on the loops (Krrivský, 1970), which takes place a little later.

Stage III - the largest spatial expansion of the flare channel; owing to the weak radiation in $H \alpha$, one speaks of the late, decay phase of the flare. The peak 'ribbon' on the loops still radiates intensely even in the coronal lines (Krivský, 1969a). This stage is probably responsible for the late phase of radio type IV, and the escape of particles and plasma will drop off.

For each of the three stages mentioned, the volumes of the following regions, according to the diagram in Figure 1, will be given: $V_{A}=$ volume of ribbon $A ; V_{B}=$ volume of ribbon $B ; V_{A}+V_{B} ; V_{C}=$ volume of 'ribbon' $C$ at the peaks of the loops; $V_{L F A}=$ volume of the foot of the loops above ribbon $A ; V_{L F B}=$ volume of the foot of the loops above ribbon $B ; V_{C}+V_{L F A}+V_{L F B}=$ volume of loops; $V_{\text {ribbons+loops }}=\left(V_{A}+V_{B}\right)+$ $+\left(V_{C}+V_{L F A}+V_{L F B}\right)$, i.e. the whole formation of the flare channel; and further

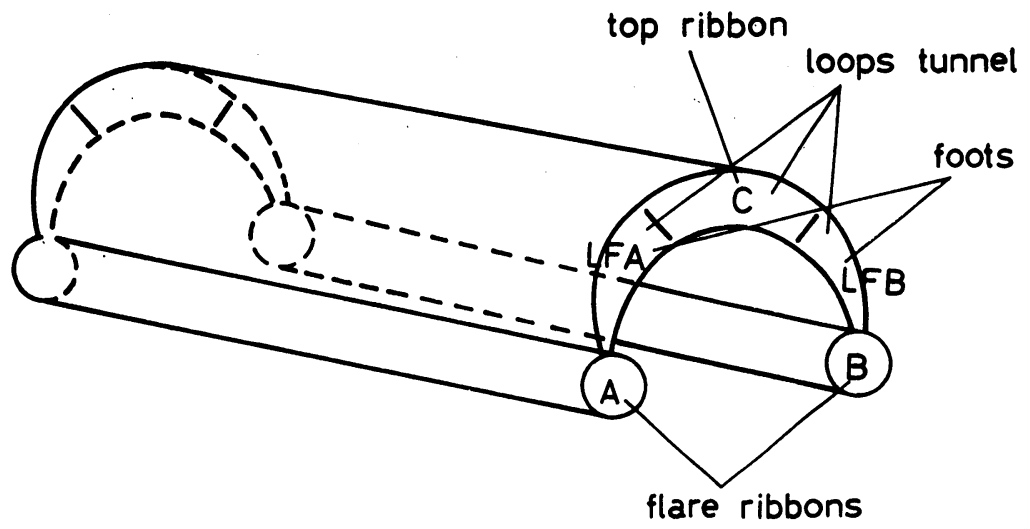

Fig. 1. Sizes of the separate parts of magnetic-channel flares. 
according tc the diagram of the Petschek model (1964) the theoretical volumes of regions III (see Figure 2), applied to the model of the magnetic channel (Krrivský, 1968, 1970), the dimensions of which have been derived from the flares measured (only for the layer III theoretical width $10^{2} \mathrm{~cm}$ is applied, Friedman and Hamberger, 1969): $V_{P \text { III } A}=$ system around the first $\mathrm{X}$-type zero point (ribbon $A$ ); $V_{P \text { III } B}=$ system around the second $\mathrm{X}$-type zero point (ribbon $B$ ); $V_{P \text { III } A}+V_{P \text { III } B}=$ volumes of both systems around zero lines.

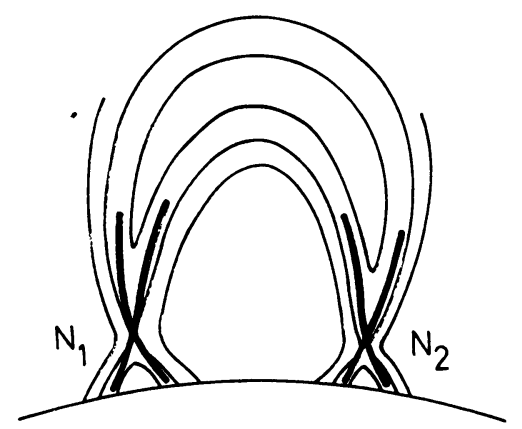

Fig.2. Vertical section of the flare channel, $N_{1}$ and $N_{2}$ are intersections of the zero lines through the plane of the vertical section. Weak lines - magnetic field lines, full lines - regions III after Petschek (1964).

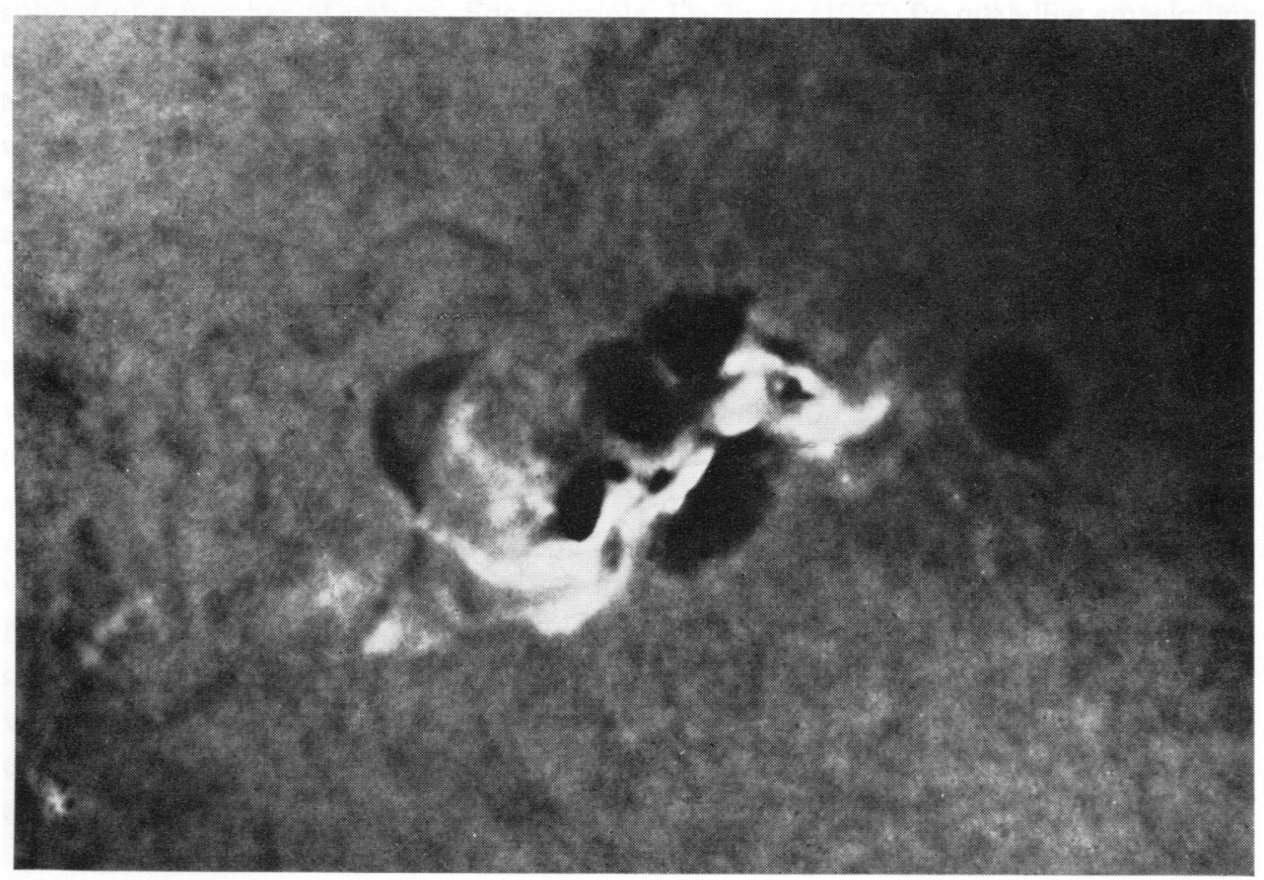

Fig. 3. Proton flare H $\alpha, 12$ July, 1961, 1023 UT (Černošice near Prague). 

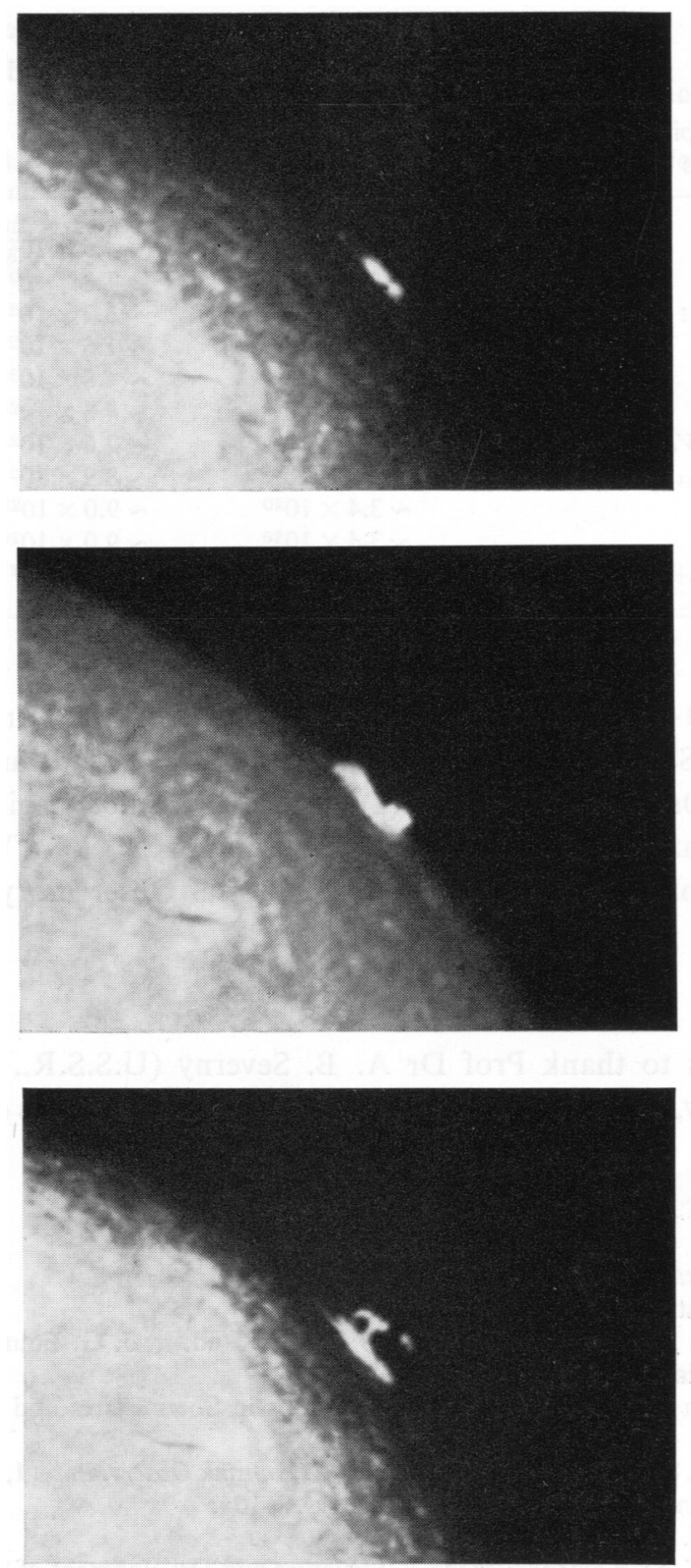

Fig. 4. Proton flare $\mathrm{H} \alpha, 18$ Nov., 1968, (a) 1030 ; (b) 10 40; (c) 1110 UT (Catania).

The volumes of the individual parts of the system of the magnetic channel of the flare according to the stages mentioned, are contained in Table I. The volumes were determined from the measurements on the photographs of two proton flares, which may be considered as being of intermediate size: (1) flare of $12 / 7 / 1961$, beginning 10:00, phase $Y 10: 20-10: 22$, maximum 10:25-10:31, end (13:03), position $7^{\circ} \mathrm{S}$, 
TABLE I

Volume characteristics of proton flares of middle size

\begin{tabular}{llll}
$\begin{array}{l}\text { Developing stages } \\
\text { volumes }\end{array}$ & $\begin{array}{l}\text { I } \\
\mathrm{cm}^{3}\end{array}$ & $\begin{array}{l}\text { II } \\
\mathrm{cm}^{3}\end{array}$ & $\begin{array}{l}\text { III } \\
\mathrm{cm}^{3}\end{array}$ \\
\hline & & & \\
$V_{A}$ & $\sim 9.6 \times 10^{26}$ & $\sim 4.5 \times 10^{27}$ & $\sim 4.8 \times 10^{27}$ \\
$V_{B}$ & $\sim 6.3 \times 10^{26}$ & $\sim 1.8 \times 10^{27}$ & $\sim 1.9 \times 10^{27}$ \\
ribbons: $V_{A}+V_{B}$ & $\sim 1.6 \times 10^{27}$ & $\sim 6.3 \times 10^{27}$ & $\sim 6.7 \times 10^{27}$ \\
$V_{C}$ & $\sim 7.0 \times 10^{26}$ & $\sim 1.6 \times 10^{27}$ & $\sim 3.0 \times 10^{27}$ \\
$V_{L F A}$ & $\sim 1.2 \times 10^{26}$ & $\sim 4.8 \times 10^{26}$ & $\sim 2.4 \times 10^{27}$ \\
$V_{L F B}$ & $\sim 1.2 \times 10^{26}$ & $\sim 4.8 \times 10^{26}$ & $\sim 2.4 \times 10^{27}$ \\
loops: $V_{C}+V_{L F A}+V_{L F B}$ & $\sim 9.4 \times 10^{26}$ & $\sim 2.6 \times 10^{27}$ & $\sim 7.8 \times 10^{27}$ \\
$V_{\text {ribbons+loops }}$ & $\sim 2.5 \times 10^{27}$ & $\sim 8.9 \times 10^{27}$ & $\sim 1.4 \times 10^{28}$ \\
$V_{P \text { III A }}$ & $\sim 3.4 \times 10^{20}$ & $\sim 9.0 \times 10^{20}$ & $\sim 1.2 \times 10^{21}$ \\
$V_{P \text { III B }}$ & $\sim 3.4 \times 10^{20}$ & $\sim 9.0 \times 10^{20}$ & $\sim 1.2 \times 10^{21}$ \\
$V_{P \text { III A }}+V_{P \text { III B }}$ & $\sim 6.8 \times 10^{20}$ & $\sim 1.8 \times 10^{21}$ & $\sim 2.4 \times 10^{21}$
\end{tabular}

$22^{\circ} \mathrm{E}$, importance $3+; \mathrm{H} \alpha$ photographs were obtained from the Crimean Astrophysical Observatory (U.S.S.R.), from Dr Otavsky's station near Prague and from the Astronomical Institute Ondřejov (Figure 3); (2) flare of 18/11/1968, beginning 10:26, phase $Y 10: 26-10: 35$, maximum $(10: 35)$, end $11: 57$, position $22^{\circ} \mathrm{N}, 87^{\circ} \mathrm{W}$, importance $2 \mathrm{~N}$. $\mathrm{H} \alpha$ photographs made at the Catania Astrophysical Observatory (Italy) were used (Figure 4).

\section{Acknowledgements}

The author wishes to thank Prof Dr A. B. Severny (U.S.S.R., Crimea) and Prof. Dr G. Godoli (Italy, Catania) who so kindly furnished $\mathrm{H} \alpha$-pictures of flares.

\section{References}

Acton, L. W.: 1968, Astrophys. J.152, 305.

Bruzek, A. and DeMastus, H. L.: 1970, Solar Phys. 12, 447.

De Jager, C.: 1967, in Electromagnetic Radiation in Space, (ed. by J. G. Emming), D. Reidel Publ. Co., Dordrecht-Holland, p. 101.

De Jager, C.: 1969, in Y. Öhman (ed.), 'Mass Motions in Solar Flares and Related Phenomena', Nobel Symp. 9, 171.

Ellison, M., McKenna, S. M. P., and Reid, J. H.: 1961, Dunsink Observ. Publ. 1, 53.

Friedman, M. and Hamberger, S. M.: 1969, Solar Phys. 8, 104.

Krrivský, L.: 1963, Bull. Astron. Inst. Czech. 14, 77.

Křivský, L.: 1968, in K. O. Kiepenheuer (ed.), 'Structure and Development of Solar Active Regions', IAU Symp. 35, 465.

Křivský, L.: 1969a, Bull. Astron. Inst. Czech. 20, 139.

Krrivský, L.: 1969b, Bull. Astron. Inst. Czech. 20, 163.

Kr̈ivský, L.: 1970, Bull. Astron. Inst. Czech. 21, 67.

Křivský, L. and Svestka, Z.: 1970, Space Research X, COSPAR XII, Prague 1969 (ed. Donahue et al.), North-Holland, Amsterdam, p. 817.

Neupert, W. M.: 1969, Ann. Rev. Astron. Astrophys. 7, 121.

Petschek, H. E.: 1964, in AAS-NASA Symp. Physics of Solar Flares 1963, Washington, p. 425.

Svestka, Z.: 1966, Space Sci. Rev. 5, 388. 
Švestka, Z.: 1970, Solar Phys. 13, 471.

Takakura, T.: 1969, Solar Phys. 6, 133.

Vaiana, G. S., Reidy, W. P., Zehnpfennig, T., Van Speybroeck, L., and Giacconi, R.: 1968, Science 161, 564.

Vaiana, G. S. and Giacconi, R.: 1969, in Plasma Instabilities in Astrophysics, (ed. by Wentzel and Tidman), Gordon and Breach, New York, p. 91.

Valní̌ek, B.: 1967, Bull. Astron. Inst. Czech. 18, 249.

Zirin, H.: 1964, Astrophys. J. 140, 1216. 